\title{
Location and color as cuing dimensions in contingent classification
}

\author{
W. R. GARNER \\ Yale University, New Haven, Connecticut
}

\begin{abstract}
The contingent classification task is one in which stimuli in a classification task are simultaneously cued by the addition of another dimension so that the classification is reduced to a discrimination of only two stimuli. The contingencies are arranged so that the cuing dimension itself provides no response information. Four experiments were carried out in which location and color were the cuing dimensions for contingent stimuli that were either letters or lines. These stimuli were considerably more difficult to perceive than those used in earlier experiments. Location provided effective contingent classification for both letters and lines, but color was ineffective with both types of stimuli. It is suggested that in order for a cuing dimension to provide effective contingent classification, its levels must in effect provide information channels, much as modalities do. Location seems to have a special status in providing such visual channels, whereas color does not. Discriminability of the levels on the cuing dimension cannot be dismissed as a factor in the different effectiveness, since the two locations were more discriminable than were the two colors. Since color was maximally discriminable, however, location still has a special status in that it can provide greater discriminability, even possibly through its ability to act as a channel.
\end{abstract}

Contingent information processing occurs whenever the attributes, features, or dimensions to be processed are determined by (i.e., contingent on) the level of some other stimulus property. Any such task includes both the cuing dimension, which specifies which properties are to be processed, and the contingent dimension, whose properties are so specified. The cuing dimension may be simultaneous with the contingent dimension, as in the present experiments, or it may precede (or precue) the contingent dimension. Garner $(1983,1985)$ has reviewed the rather limited literature on this topic, including contingent stimulus-response relations (e.g., Shaffer, 1965) and contingent identification (e.g., Biederman, 1972). In both of these contingent tasks, the cuing dimension not only specifies something about the perceptual properties to be processed, but also gives direct response information.

In most such experiments, when the cuing dimension precedes (i.e., precues) the occurrence of the contingent dimension, the primary purpose is in fact to investigate partial response preparation. This has been the concern of Miller $(1982,1983,1985)$ and Reeve and Proctor $(1984,1985)$. The precuing technique has been used for a variety of other purposes as well (see LaBerge, Van Gelder, \& Yellott, 1970; Posner, 1978). However, the intentional or coincidental direct cuing of the response in these experiments limits their ability to investigate purely perceptual relations between the cuing and the contingent stimulus dimensions.

This research was supported by National Institute of Health Grant MH 14229 to Yale University. Carolyn Paul carried out all of the experiments, helped design them, and did most of the data analysis. Her invaluable assistance is gratefully acknowledged. Requests for reprints should be sent to W. R. Garner, Department of Psychology, Box 11-A Yale Station, New Haven, CT 06520-7447.

\section{Contingent Classification}

The contingent classification task is a form of contingent information processing in which the cuing dimension provides no direct response information. The basic task is one of classification, in which more than one stimulus is assigned a single response. The cuing dimension provides a contingent relation so that its level on a particular trial specifies a smaller number of possible stimuli, but does not specify which response should be used. In a simple example, suppose that the contingent stimuli are $A$ or $B / C$ or $D$, with the slash separating the pairs of stimuli that are to be given each response. If a cuing dimension of color is added, a red stimulus might indicate that $\mathrm{A}$ or $\mathrm{C}$ can occur on that trial, whereas a green stimulus would indicate that B or D can occur. Since both of these cued pairs of stimuli require both responses, the cuing dimension of color has not given any information about the response to be given.

With a 4:2 mapping of stimuli to responses for the basic classification task, the addition of a cuing dimension essentially changes the classification task to one of discrimination, with a 2:2 mapping of contingent stimuli to responses on a random trial-by-trial basis. With appropriate sets of contingent stimuli, the basic classification task can be made hard or easy. Ordinarily, we would expect that the addition of a cuing dimension to an easy classification task would provide little or no improvement, and in the limiting case in which the classification task allows perfect selective attention to the property defining the classification, there can be no improvement. Furthermore, at least with a hard classification task, the cuing dimension can be assigned so that the resultant discriminations are themselves hard or easy. Thus the contingent classification task can be useful in understanding relations be- 
tween cuing and contingent dimensions without the confound that occurs when the cuing dimension also gives direct response information.

Garner (1985) carried out a series of experiments with the stimuli $\mathrm{C}, \mathrm{O}, \mathrm{L}$, and $\mathrm{T}$, using reaction time (RT) as the measure of performance. This set of stimuli allows an easy classification, $\mathrm{CO} / \mathrm{LT}$, based on the curvedstraight distinction, and two hard classifications, CL/OT and CT/OL. With the easy classification, all cued discrimination possibilities are also easy, since they allow the curved-straight property to be used. With hard classifications, however, the cuing can be done so as to provide easy discriminations or hard discriminations. To illustrate with the CL/OT task, if the cuing dimension separates the stimuli into pairs of $\mathrm{C} / \mathrm{O}$ and $\mathrm{L} / \mathrm{T}$, the discriminations are hard, whereas if the cuing dimension separates the stimuli into pairs of $\mathrm{C} / \mathrm{T}$ and $\mathrm{L} / \mathrm{O}$, the discriminations are easy.

The results of Garner's (1985) experiment showed that color as a cuing dimension provided no improvement in performance. Lateral location as a cuing dimension showed a slight improvement, but only when the resultant discrimination tasks were easy. Since it was possible that the random alternation of what could be processed as discriminations might have made the task much more difficult, a 500-msec precuing task was also used, and in this case the precuing was almost completely effective in changing performance in the precued contingent task so that RTs were equivalent to those obtained with the discrimination tasks used alone.

In Garner's (1985) discussion of why so little use was made of the contingent relation, several suggestions were offered, including the possibility that subjects simply allocated all of their attention to the classification task alone, not being convinced that some sharing of attention would provide as much of a gain as could be lost from sharing attention. One specific suggestion concerning how to change such strategies was to make the contingent stimuli much more difficult to discriminate or classify. Such a change would make it more likely that subjects would allocate enough attention to the cuing dimension that there would be an improvement in performance by its use.

\section{Purpose of Experiments}

The specific purpose of the present experiments was to determine whether contingent classification would be a more effective procedure if the contingent stimuli were more difficult to perceive (i.e., to classify or to discriminate). Two types of contingent stimuli were used. The first type is the same set of $\mathrm{C}, \mathrm{O}, \mathrm{L}, \mathrm{T}$ stimuli that had been used by Garner (1985), except that they were degraded in several ways to make them much more difficult to perceive. In the earlier experiments, the letters were relatively large, bold, and, in general, very easy to perceive. They were not in the present experiments. The second type of contingent stimuli was the set of four lines previously used by Lasaga and Garner (1983): vertical (V), horizontal (H), left diagonal (L), and right diagonal
(R). Not only were these stimuli somewhat more difficult to classify and discriminate than were the bold letters used by Garner (1985), but they were simpler in nature. It seemed possible that the combination of simpler, yet more difficult, stimuli would allow or encourage greater allocation of attention to the cuing dimension. These stimuli also provided one easy (VH/RL) and two hard (VR/HL and HR/VL) classification tasks, as with the letter stimuli, but the hard classification tasks did not provide two sets of discrimination tasks of unequal difficulty.

The same cuing dimensions previously used by Garner (1985) were used in these experiments: location and color. Four experiments were produced by the crossing of the two cuing dimensions of location and color with the two types of contingent stimuli, letters and lines. Experiments 1 and 2 used location cuing with letters and lines, respectively; Experiments 3 and 4 used color cuing with letters and lines, respectively.

\section{EXPERIMENT 1: LOCATION-CUED LETTERS}

\section{Method}

The methods of all four experiments were identical, except for the specific stimuli, tasks, and cuing dimensions, and the resultant changes in reminder cards. Therefore, a complete description of methodology is given for Experiment 1, and changes are indicated for Experiments 2-4.

Stimuli. The stimuli were the letters $\mathrm{C}, \mathrm{O}, \mathrm{L}$, and $\mathrm{T}$, which were presented at vertical center to the left or right of horizontal center. Compared with the same letters used by Garner (1985), these letters were degraded in three ways. First, smaller letters were used (Letraset 430, 16-pt Folio Light). These letters are approximately $3.5 \mathrm{~mm}$ (visual angle or $\mathrm{VA}=.16^{\circ}$ ) high, with a line thickness of about $0.4 \mathrm{~mm}$. These are about half the size and a third the thickness of those used previously. Second, the letters were masked by placing an 8.0-mm $\left(\mathrm{VA}=.36^{\circ}\right)$ square gray patch (Pantone 431A) over the letters. Third, the general luminance was reduced from $17.1 \mathrm{~cd} / \mathrm{m}^{2}$ to $10.3 \mathrm{~cd} / \mathrm{m}^{2}$. Simply put, these letters were very difficult to see, although, as the error rates attest, they could be seen well enough for identification. The stimuli were presented in a three-field Scientific Prototype tachistoscope, for which $12.7 \times 17.8 \mathrm{~cm}$ cards were used to display stimulus materials in all three fields, and were viewed binocularly at a distance of $127 \mathrm{~cm}$.

Tasks. Seven tasks were used, and these were of two basic types, classification and discrimination. Three classification tasks were used: a control classification task, CL/OT, in which the slash separates the stimuli to be given different responses; and two contingent classification tasks, $\mathrm{C} / \mathrm{T}, \mathrm{L} / \mathrm{O}$ and $\mathrm{C} / \mathrm{O}, \mathrm{L} / \mathrm{T}$, in which the slash separates different responses and the comma separates the two cued components of the contingent task. For example, C/T,L/O would mean that $C$ or $T$ always appeared on one side and $L$ or $O$ on the other. The control classification is a hard one, based on the previous research, and the $\mathrm{C} / \mathrm{T}, \mathrm{L} / \mathrm{O}$ contingent task provides two easy discriminations, whereas $\mathrm{C} / \mathrm{O}, \mathrm{L} / \mathrm{T}$ provides two hard discriminations.

Four discrimination tasks were used, which involved the same four cued discriminations used in contingent classification: $C / T$, $\mathrm{L} / \mathrm{O}, \mathrm{C} / \mathrm{O}$, and $\mathrm{L} / \mathrm{T}$. Thus direct comparisons of task difficulty between the contingent tasks and their equivalent discrimination tasks are possible. In analyzing the data, the two easy and two hard discriminations are combined into single means. Not only are the easy and hard discriminations equivalent within themselves in difficulty, but since all stimuli were presented an equal number of times, 
statistical comparisons between discrimination and classification tasks are comparable in terms of total number of trials.

The centers of the letters appeared $11.25 \mathrm{~mm}\left(\mathrm{VA}=.51^{\circ}\right)$ to the right or left of horizontal center. For all tasks a single letter appeared as the stimulus on a single trial. In control classification and in discrimination tasks, the particular letters appeared in a random order, and their location was also random from trial to trial, so that all letters used in a task appeared randomly at both locations. In the two contingent tasks, the letters and locations were random from trial to trial, but whenever one of a particular two letters occurred (e.g., C or T), it was always on only the right or only the left. The other two letters always appeared at the other location. Thus lateral location served as the cuing dimension in the contingent classification tasks, since once the location was specified, so also was the particular pair of letters that could occur on that trial. Although location varied in the control classification and discrimination tasks, the variation was random for all letters used and thus could provide no additional information.

To summarize, in discrimination and control classification, both letters and locations varied randomly, with all letters used appearing equally often at both locations; in contingent classification, locations and letters varied randomly, but the letters were contingent on the locations, so that only two letters appeared at each location. This contingent relation is the only difference between control and contingent classification; that is, all letters appeared randomly in both tasks, and locations were random in both tasks, but in the contingent tasks there was a correlation (contingency) between location and letters.

The randomization of both letters and locations was constrained so that both locations, all letters, and the appropriate combinations of locations and letters occurred equally often.

Reminder cards. At all times before and after an actual experimental trial, a card was visible with a fixation dot at its center, although subjects were not required to maintain strict fixation. Letters $\left(5 \mathrm{~mm}\right.$ high) located at horizontal center $20 \mathrm{~mm}\left(\mathrm{VA}=.90^{\circ}\right)$ above and below the vertical center of the card indicated the appropriate response assignments, and the actual response assignments (up or down) were counterbalanced across subjects.

For discrimination tasks, the two letters on the reminder card were centered horizontally, above and below the fixation dot. In control classification, two additional letters necessary to specify the responses were located $11 \mathrm{~mm}$ higher or lower, center to center, than the reminder letters used in discrimination tasks. This arrangement was necessary to avoid providing any location information. With contingent classification, the four letters indicating response assignment were located above and below vertical center, as in the discrimination tasks, but were located horizontally to the left and right to correspond exactly to the possible locations of the stimulus letters that could occur on either side. The bottoms of these letters were $15.75 \mathrm{~mm}$ above the tops of the stimulus letters $\left(V A=.71^{\circ}\right.$ ) and never appeared when the stimulus letter appeared, so there is no reason to expect any interference from the reminder letters on the stimulus letters.

Trials. A single trial commenced when the subject pushed a button switch held in the left hand. At this time the reminder card remained on for $400 \mathrm{msec}$; the field then went blank at the same luminance for $100 \mathrm{msec}$; and then the single stimulus appeared and remained for 1,000 msec. The subject responded by moving a small switch lever (mounted under the tachistoscope) up or down according to the specific response assignment indicated by the reminder card. A computer recorded the response and the RT to the nearest millisecond and also randomized the stimulus presentation orders. The total time for a single trial was about $6 \mathrm{sec}$.

Sessions. Each session of about 50 min required the subjects to perform all seven experimental tasks. For the classification tasks, each stimulus was presented 24 times for a total of 96 trials. In control classification, with locations and letters varying randomly with no contingent constraint, each stimulus letter was used 12 times at each location. With contingent classification, since each letter occurred at only one location, each letter was used 24 times at each location. In either case, for purposes of initial analysis, a block of trials was considered to include each letter-location combination. With discrimination tasks, each stimulus letter was used 24 times, 12 at each location, and also for purposes of initial analysis, a block of trials was considered to include each stimulus-location combination. Thus 480 trials were run in a single session, plus 4-8 practice trials before each new task. More practice trials were allowed if the subject requested them.

All subjects were run for three sessions, but the data of the first session were discarded as practice, and only the data of the last two sessions were used. The subjects took part in only one session in a day, except on rare occasions, in which case at least $1 \mathrm{~h}$ separated the sessions.

Experimental design. The seven tasks and 7 subjects were arranged in a Latin square for counterbalancing purposes. The same Latin square was used for each session and was repeated with a second group of 7 subjects. The Latin square ensured that no task preceded or followed another one more than twice, and also distributed the discrimination and classification tasks for each subject as evenly as possible through the seven task orders.

Subjects. The 14 subjects, recruited from Yale students and staff, were paid for participation. Some had had previous experience with this type of research and some were naive. All had normal or corrected-to-normal vision.

Data analysis. Error rates were small for this and the other three experiments, with overall rates ranging from $3.0 \%$ to $5.3 \%$. Furthermore, within each experiment the product-moment correlation across conditions between error rates and RTs was positive, ranging from .57 to .99 . Thus there is no evidence of a speed-accuracy tradeoff. That fact, plus the small number of errors, means that little can be gained by further analysis of errors, so errors will not be discussed in this or the other experiments.

The primary dependent variable, therefore, is RT. For each block of stimulus-location trials for each subject, a median RT was computer calculated (correct responses only), the median being used to minimize the effect of outliers. All further analyses were done with means of these original median scores. Analyses of variance (ANOVAs) were then used for additional analyses, with various contrast tests (Winer, 1971 , p. $170 \mathrm{ff}$.) to address specific questions. A significance level of $p<.01$ was used unless otherwise specified.

Preliminary experiment. Because of the substantial change in the nature of the letter stimuli from the previous research, a preliminary experiment was run to establish that the hard and easy classification tasks still differed as they did in Garner (1985). An experiment was run in which the easy task, CO/LT, was compared with the hard task used in this experiment, CL/OT, with only these two conditions run on an alternating basis three times each, 96 trials each time, with 8 subjects. The overall mean for the hard task was $765 \mathrm{msec}$, and that for the easy task was $632 \mathrm{msec}$. This difference is not only clearly significant in the expected direction [ $t(7)$ $=8.48$ ], but is larger than that previously obtained (Garner, 1985, Experiment 1, where the RTs were 373 msec for the easy task and $454 \mathrm{msec}$ for the hard task). The increased difference could easily be due to the greater range of RT values. Nevertheless, the preliminary experiment did establish that the control classification task used in the experiment was hard.

\section{Results}

A preliminary analysis was performed to determine whether the left or right location of the stimuli had any effect on RT. The overall mean for all stimuli on the left was $633 \mathrm{msec}$, and for those on the right it was $626 \mathrm{msec}$. 
Table 1

Experiment 1: Reaction Times for Control Classification, Contingent Classification Tasks Cued by Location, and the Equivalent Discrimination Tasks, Using Letter Stimuli

\begin{tabular}{lcc}
\hline Task Type & Specific Task & Mean RT \\
\hline Classification: & CL/OT & 706 \\
Control & C/T,L/O & 643 \\
Contingent & C/O,L/T & 681 \\
Contingent & & \\
Discrimination: & C/T and L/O & 554 \\
& C/O and L/T & 633 \\
\hline
\end{tabular}

Note-The slash separates the stimuli to be given different responses; the comma separates the two components of the contingent classification tasks; and RTs (in milliseconds) are averaged for the pairs of discrimination tasks that correspond to the two equivalent contingent tasks.

This is a nonsignificant difference, so the left-right position is not a factor that needs to be considered.

The data from this experiment are displayed in Table 1, with the data for the discrimination tasks averaged to correspond to the equivalent contingent tasks as explained above. An initial ANOVA was carried out first, and it showed significant effects of tasks $[F(4,52)=39.44]$. A contrast between the three classification tasks and the two pairs of discrimination tasks was also significant $[F(1,52)$ $=97.24]$. This effect is evident from the tabled data, since the lowest RT for any classification task $(643 \mathrm{msec})$ was higher than the greater RT for the discrimination tasks $(633 \mathrm{msec})$. However, for the questions involved in this experiment, analyses within each type of task are more important.

Classification tasks. The control classification was slower than either of the contingent tasks (706 vs. 643 and $681 \mathrm{msec}$ ). A contrast between the two types of classification was significant $[F(1,52)=15.47]$. Further contrasts between the control and easy contingent tasks showed significance for the easy contingent task $(\mathrm{C} / \mathrm{T}, \mathrm{L} / \mathrm{O})[F(1,52)=23.50]$, but only marginal significance for the harder contingent task $(\mathrm{C} / \mathrm{O}, \mathrm{L} / \mathrm{T})$ $[F(1,52)=3.86, p \approx .06]$. However, since the difference is in the expected direction, this result can also be accepted as significant. A contrast between the two contingent tasks, with a difference of $38 \mathrm{msec}$, was also significant $[F(1,52)=8.31]$. Thus there is clear evidence that contingent processing occurred, and that these two contingent tasks differed in performance, as they should if contingent processing changes the classification task to a randomly alternating discrimination task that can be hard or easy.

Discrimination tasks. The hard discrimination tasks, $\mathrm{C} / \mathrm{O}$ and $\mathrm{L} / \mathrm{T}$, gave a mean RT of $633 \mathrm{msec}$, and the two easy tasks, C/T and L/O, gave a mean RT of 554 msec. This difference of $79 \mathrm{msec}$ was significant, as expected $[F(1,52)=36.75]$. However, each contingent classification was slower than its equivalent discrimination tasks. For the easy task, the difference was $89 \mathrm{msec}[F(1,52)$ $=46.51]$; for the difficult task, the difference was
$48 \mathrm{msec}[F(1,52)=13.25]$. Both the comparisons between contingent tasks and those between discrimination tasks, as well as the comparisons between each contingent task and its comparable discrimination tasks show an interaction that is significant $[F(1,52)=5.05$, $p<.05]$.

\section{Discussion}

With the more difficult letters used here, there is unequivocal evidence that the use of a simultaneous cuing dimension to provide a contingent classification task is effective in substantially improving performance. And the fact that the contingent tasks with hard and easy discriminations differed according to these differences in ease of discrimination is clear evidence that the contingent classification task does change classification into discriminations between two stimuli. Nevertheless, the improvement with the contingent task did not provide RTs comparable to those obtained with the discrimination tasks used alone. Thus there is room for considerably more improvement in subjects' performance than is shown here. Furthermore, the substantial interaction between the hard and easy discrimination tasks and their equivalent contingent tasks, with the difference between the contingent tasks being about half that of the discrimination tasks, suggests that the contingent task still does not completely alter the classification task to one of randomly alternating discrimination tasks.

\section{EXPERIMENT 2: LOCATION-CUED LINES}

\section{Method}

In this experiment all aspects of method were identical to those of Experiment 1 except for the stimuli, the reminder cards, and the specific tasks.

Stimuli. The stimuli were four lines, vertical $(V)$, horizontal (H), left diagonal (L), and right diagonal (R). The two diagonal lines were rotated $45^{\circ}$ left or right of vertical. These stimuli correspond to those used previously by Lasaga and Garner (1983), and were black, drawn with a felt pen, $8 \mathrm{~mm}\left(\mathrm{VA}=.36^{\circ}\right)$ long and $0.5 \mathrm{~mm}$ wide. Background luminance was $17.1 \mathrm{~cd} / \mathrm{m}^{2}$. As in Experiment 1, the lines were vertically centered $11.25 \mathrm{~mm}$ to the right or left of horizontal center. With both control classification and discrimination tasks, the lines appeared in random sequence at either location, also randomly. With the two contingent tasks, two lines were assigned to each location, so that location and stimulus lines were both random, but with the contingency that only two lines could appear at each location.

Reminder cards. With discrimination tasks, the lines on the reminder card were centered above and below the fixation dot. With control classification, an additional line was placed above and below these lines, as in Experiment 1. With contingent classification, the lines were located above and below the actual possible locations of the stimuli. All dimensions for locations on the reminder card are the same as in Experiment 1. The lines themselves were $6 \mathrm{~mm}\left(\mathrm{VA}=.27^{\circ}\right)$ long.

Tasks. The specific tasks were: VR/HL for control classification, $V / L, R / H$ and $V / H, R / L$ for the two contingent classifications, and $V / L, R / H, V / H, R / L$ for the equivalent discrimination tasks. Unlike the contingent classifications using letters in Experiment 1, these two contingent classifications do not provide a clear distinc- 
tion between hard and easy discriminations. In Lasaga and Garner (1983), with discrimination tasks, $\mathrm{R} / \mathrm{L}$ produced slower $\mathrm{RT}$ s than all the others, but no further differences existed.

Preliminary experiments. Two preliminary experiments were run, each comparing two classification tasks, with three alternations of the tasks, 96 trials per task each time, and with 8 subjects. The first one established that the line stimuli were more difficult than the bold letters used by Garner (1985), with random locations in both cases. The mean RT for VR/HL was $509 \mathrm{msec}$, and that for CL/OT was $439 \mathrm{msec}$. This difference of $70 \mathrm{msec}$ was significant $[t(7)=4.92]$. Although the line stimuli are less difficult than the degraded letters used in Experiment 1, they are definitely more difficult than the letters used in the previous research (Garner, 1985).

The second preliminary experiment was to determine that the use of random locations of the lines did not alter the relation between easy and hard classifications. The mean RT for VH/RL was $442 \mathrm{msec}$, and that for VR/HL was $540 \mathrm{msec}$. This difference of $98 \mathrm{msec}$ was significant $[t(7)=4.96]$, and corresponded very closely to the difference of 92 msec obtained by Lasaga and Garner (1983). Thus the two preliminary experiments established that line stimuli are more difficult (but also simpler) than the previously used bold letters, and that the particular classification task being used was in fact a hard one compared with the previously found easy one.

\section{Results}

A preliminary analysis was performed to determine whether the left or right location of the stimuli had any effect on RT. The overall mean for all stimuli on the left was $438 \mathrm{msec}$, and for all on the right it was $431 \mathrm{msec}$. This is a nonsignificant difference, and location was disregarded in all further analyses.

The data from this experiment are displayed in Table 2, again with the discrimination tasks averaged to correspond to the equivalent contingent tasks. The overall result is similar to that from Experiment 1, with the contingent classifications being faster than the control classification, but not as fast as the discrimination tasks.

The initial ANOVA established that there were significant effects of tasks $[F(4,52)=82.62]$, and a contrast between the three classification tasks and the two pairs of discrimination tasks was also significant $[F(1,52)=$ 286.49]. Further analyses are within each of the two types of task.

Table 2

Experiment 2: Reaction Times for Control Classification, Contingent Classification Tasks Cued by Location, and the Equivalent Discrimination Tasks, Using Line Stimuli

\begin{tabular}{ccc}
\hline Task Type & Specific Task & Mean RT \\
\hline Classification: & VR/HL & \\
Control & V/L,R/H & 537 \\
Contingent & V/H,R/L & 479 \\
Contingent & & 478 \\
Discrimination: & V/L and R/H & \\
& V/H and R/L & 382 \\
&
\end{tabular}

Note-The slash separates the stimuli to be given different responses; the comma separates the two components of the contingent classification tasks; and RTs (in milliseconds) are averaged for the pairs of discrimination tasks that correspond to the two equivalent contingent tasks. $\mathrm{V}=$ vertical; $\mathrm{H}=$ horizontal; $\mathbf{R}=$ right diagonal $\mathrm{L}=$ left diagonal.
Classification tasks. The control classification was slower than either of the contingent tasks (537 vs. 479 and $478 \mathrm{msec}$ ), which were themselves essentially identical. For $\mathrm{V} / \mathrm{L}, \mathrm{R} / \mathrm{H}, F(1,52)=31.70$, and for $\mathrm{V} / \mathrm{H}, \mathrm{R} / \mathrm{L}$, $F(1,52)=32.89$. Thus there is clear evidence that contingent processing occurred and that performance for the two contingent tasks did not differ.

Discrimination tasks. The difference of $9 \mathrm{msec}$ for performance on the two pairs of discrimination tasks was not significant. However, since previous research (Lasaga \& Garner, 1983) showed the $\mathrm{R} / \mathrm{L}$ task to be more difficult than the other discrimination task, an ANOVA was carried out in which the four discrimination tasks were not averaged into two pairs. This analysis showed mean RTs of $410,378,385$, and $373 \mathrm{msec}$ for the $\mathrm{R} / \mathrm{L}, \mathrm{R} / \mathrm{H}, \mathrm{V} / \mathrm{L}$, and $\mathrm{V} / \mathrm{H}$ tasks, respectively. A contrast between $\mathrm{R} / \mathrm{L}$ and the other three tasks was significant $[F(1,78)=12.71]$, but none of the other three tasks differed from one another. This result completely replicates the previous finding by Lasaga and Garner (1983).

In order to determine whether this greater difficulty was reflected in the contingent tasks, an additional analysis was done in which each of the two equivalent discrimination tasks for each contingent classification was treated separately, as were the separate discrimination tasks. For the $\mathrm{V} / \mathrm{H}, \mathrm{R} / \mathrm{L}$ task, the two means were 462 and $495 \mathrm{msec}$, respectively, and this difference was significant $[F(1,91)$ $=5.94, p<.05]$. The two means for the $\mathrm{V} / \mathrm{L}, \mathrm{R} / \mathrm{H}$ task were 490 and $469 \mathrm{msec}$, respectively, and this difference was not significant. Thus the differences in the discrimination tasks are reflected in the equivalent discrimination tasks in the contingent classifications.

Despite the fact that contingent processing did occur, once again each contingent classification was slower than its equivalent discrimination tasks. For the $\mathrm{V} / \mathrm{L}, \mathrm{R} / \mathrm{H}$ task, the difference was $97 \mathrm{msec}[F(1,52)=91.41]$. For the $\mathrm{V} / \mathrm{H}, \mathrm{R} / \mathrm{L}$ task, the difference was $87 \mathrm{msec}[F(1,52)=$ 72.28].

\section{Discussion}

As in Experiment 1, these line stimuli show clear evidence that simultaneous location cuing improves performance in the contingent classification task. In this experiment, there was no difference in performance for the two contingent tasks themselves. Yet more detailed analysis showed that the R/L task was more difficult than the other discrimination tasks, and this difference in difficulty was evident within the one contingent task in which the $R / L$ equivalent discrimination task occurred. It is therefore reasonable to assume that the contingent task does change the classification task to one of alternating discrimination tasks. Nevertheless, the contingent classification does not produce processing speeds comparable to those obtained with the discrimination tasks used singly. Thus the cuing information provided by location differences is not completely effective. 


\section{EXPERIMENT 3: COLOR-CUED LETTERS}

\section{Method}

In this experiment all aspects of method were the same as in Experiment 1 , except for the stimuli and cuing dimension, reminder cards, specific tasks, and subjects.

Stimuli. The cuing dimension for this experiment was color, and it was presented by having the entire stimulus card colored. The two colors used were a highly saturated red (Pantone 032-A, Munsell equivalent 5R 5.5/14) and a green (Pantone 347-A, Munsell equivalent 2.5G 5.5/12). In control classification and discrimination tasks, both colors and letters varied randomly. In contingent classification, both colors and letters again varied randomly, but with the constraint that only two letters were to appear with each of the colors.

The actual letter stimuli $(\mathrm{C}, \mathrm{O}, \mathrm{L}, \mathrm{T})$ were identical to those used in Experiment 1, except for three differences. The letters were placed directly over the colored card, thus reducing the contrast even more. In compensation, the luminance was increased to $17.1 \mathrm{~cd} / \mathrm{m}^{2}$. The stimulus letters always appeared in the center of the stimulus card.

Reminder cards. All letters on the reminder cards were black. In discrimination tasks, the reminder letters that indicated response assignments were centered horizontally, above and below fixation. In control classification, two letters were above and two below fixation to specify the response. These letters were separated horizontally by $25 \mathrm{~mm}\left(\mathrm{VA}=1.13^{\circ}\right)$, center to center. The same location of the reminder letters was used for the contingent tasks, but, in addition, an $8.0-\mathrm{mm}\left(\mathrm{VA}=.36^{\circ}\right)$ square of the appropriate color was located above the upper letters or below the lower letters ( $7.0 \mathrm{~mm}$ from the nearest edge), to indicate the contingent relations.

One additional difference from Experiment 1 is that in the $100 \mathrm{msec}$ after the reminder card went off and before the stimulus appeared, a gray field of the same 5.5 Munsell value appeared so that the stimulus would not suddenly change from white to the darker color.

Tasks. The same tasks were used as in Experiment 1: CL/OT for the control classification, $\mathrm{C} / \mathrm{T}, \mathrm{L} / \mathrm{O}$ and $\mathrm{C} / \mathrm{O}, \mathrm{L} / \mathrm{T}$ for the two contingent tasks, and $\mathrm{C} / \mathrm{T}, \mathrm{L} / \mathrm{O}, \mathrm{C} / \mathrm{O}$, and $\mathrm{L} / \mathrm{T}$ for the discrimination tasks. As before, data for the discrimination tasks were averaged into the two pairs indicated by the contingent classification tasks.

Subjects. Seven subjects were used, and they were given a simple color test in which they were required to select a good green and a good red from a random array of colors. All subjects were able to pass this test.

\section{Results}

Classification tasks. The data from this experiment are displayed in Table 3, and the initial ANOVA showed that there were significant effects of tasks $[F(4,24)=27.17]$. However, as is evident from the displayed data and the appropriate contrast, most of this significant effect was due to the differences between the classification and discrimination tasks $[F(1,24)=91.32]$. There were no significant differences among any of the three classification tasks (all $F s<1.0$ ). Thus there is no evidence that any effective contingent processing occurred.

Discrimination tasks. The hard discrimination tasks, $\mathrm{C} / \mathrm{O}$ and $\mathrm{L} / \mathrm{T}$, gave a mean RT of $587 \mathrm{msec}$, as compared with that of $500 \mathrm{msec}$ for the easy tasks. This difference of $87 \mathrm{msec}$ is significant $[F(1,24)=17.00]$ and corresponds very closely to the equivalent difference of $79 \mathrm{msec}$ for the same stimuli in Experiment 1 . Each pair
Table 3

Experiment 3: Reaction Times for Control Classification, Contingent Classification Tasks Cued by Color, and the Equivalent Discrimination Tasks Using Letter Stimuli

\begin{tabular}{lcc}
\hline Task Type & Specific Task & Mean RT \\
\hline Classification: & CL/OT & 678 \\
Control & C/T,L/O & 666 \\
Contingent & C/O,L/T & 677 \\
Contingent & & \\
Discrimination: & C/T and L/O & 500 \\
& C/O and L/T & 587 \\
\hline
\end{tabular}

Note-The slash separates the stimuli to be given different responses; the comma separates the two components of the contingent classification tasks; and RTs (in milliseconds) are averaged for the pairs of discrimination tasks that correspond to the two equivalent contingent tasks.

of discrimination tasks was also significantly faster than its equivalent contingent classification $[F(1,24)=62.03$ for the easy discrimination, and $F(1,24)=18.33$ for the hard discrimination]. Since there was no difference between the two contingent classification tasks, but there was for the equivalent discrimination tasks, the interaction is also significant $[F(1,24)=8.53]$.

\section{Discussion}

The use of color as the cuing dimension with the degraded letters gave no evidence of effective contingent processing, unlike the use of location as the cuing dimension in Experiment 1. That the differences in the contingent stimuli themselves is not responsible is clear from the results for the discrimination tasks, in which the difference between hard and easy discriminations was about the same as had been obtained in Experiment 1, and with reasonably comparable absolute times. Furthermore, these discrimination tasks were so much faster than any of the classification tasks that there was more than adequate capability of improvement in performance. In addition, the red and green background colors were highly saturated and as different as any two colors can be. Thus the lack of effective contingent processing cannot be due to the nature of the contingent letter stimuli, and is not likely due to a low discriminability between the two levels of the cuing dimension. Rather, it is probably due to the nature of color itself as a cuing dimension.

\section{EXPERIMENT 4: COLOR-CUED LINES}

\section{Method}

In this experiment all aspects of method were the same as in Experiment 1 , except for the stimuli and cuing dimension, reminder cards, specific tasks, and subjects. As in Experiment 3, the 7 subjects took part in a screening color test.

Stimuli. The stimuli were the same four lines used in Experiment 2, the vertical, horizontal, and two diagonals. The dimensions were the same, and the background luminance was $17.1 \mathrm{~cd} / \mathrm{m}^{2}$. These stimuli were presented in the center of the visual field.

The cuing dimension was color, and the lines were drawn with a felt pen in the appropriate color and seen against a white background. The colors were red and green, and these colors were matched as closely as possible to those used in Experiment 3. 
Reminder cards. All lines on the reminder cards were placed above or below the fixation point. For discrimination tasks one line was above and one below the point, and these were black. For control classification, black lines were also used, with two lines vertically aligned above and two below the fixation point, as in control classification of Experiment 2. These lines were also black. For the contingent tasks, the same vertical alignment of the lines was used, but one above and one below were green and the other two were red, to provide the contingent assignments. Even though the lines on the reminder cards were black for all discrimination and the control classification tasks, the actual stimuli were always colored, with color varying randomly for these conditions.

Tasks. The same specific tasks were used as in Experiment 2: VR/HL for control classification, $V / L, R / H$ and $V / H, R / L$ for the two contingent classifications, and the equivalent discrimination tasks, $V / L, R / H, V / H$, and $R / L$. Data for the discrimination tasks were averaged into the two pairs indicated by the contingent classification tasks.

\section{Results}

Classification tasks. The data from this experiment are displayed in Table 4, and an initial ANOVA showed that there were significant effects of tasks $[F(4,24)=17.97]$. However, as is evident in the data and the appropriate contrast, most of this effect was due to the difference between the classification and discrimination tasks $[F(1,24)$ $=68.47]$. There were no significant differences among any of the three classification tasks (largest $F=1.18$ ). Thus, as in Experiment 3, there is no evidence that any effective contingent processing occurred.

Discrimination tasks. The difference of $25 \mathrm{msec}$ between the two pairs of discrimination tasks was not significant. However, as in Experiment 2, an additional ANOVA was carried out in which the four discrimination tasks were not averaged into two pairs. This analysis showed mean RTs of 409, 368, 376, and 385 msec for the $R / L, R / H, V / L$, and $V / H$ tasks, respectively. A contrast between $R / L$ and the other three tasks was significant $[F(1,36)=4.81, p<.05]$, but none of the other three differed from one another. This slower RT for the $\mathrm{R} / \mathrm{L}$ task replicates the finding of Experiment 2, as well as that of Lasaga and Garner (1983).

Although there was no evidence of effective contingent processing in this experiment, an additional analysis was

Table 4

Experiment 4: Reaction Times for Control Classification, Contingent Classification Tasks Cued by Color, and the Equivalent Discrimination Tasks, Using Line Stimuli

\begin{tabular}{lcc}
\hline Task Type & Specific Task & Mean RT \\
\hline Classification: & VR/HL & \\
Control & V/L,R/H & 475 \\
Contingent & V/H,R/L & 474 \\
Contingent & & 493 \\
Discrimination: & V/L and R/H & \\
& V/H and R/L & 372 \\
\hline
\end{tabular}

Note-The slash separates the stimuli to be given different responses; the comma separates the two components of the contingent classification tasks; and RTs (in milliseconds) are averaged for the pairs of discrimination tasks that correspond to the two equivalent contingent tasks. $\mathrm{V}=$ vertical; $\mathbf{H}=$ horizontal; $\mathbf{R}=$ right diagonal; $\mathbf{L}=$ left diagonal. done in which each of the two equivalent discrimination tasks for each contingent classification was treated separately, along with separate discrimination tasks. For the $V / H, R / L$ task, the two means were 491 and $495 \mathrm{msec}$, respectively; for the $\mathrm{V} / \mathrm{L}, \mathrm{R} / \mathrm{H}$ task, both means were $474 \mathrm{msec}$. Clearly there were no significant differences between performance on equivalent discrimination tasks within either of the contingent classification tasks. Thus the lack of effective contingent processing is reflected in that there were no differences between the equivalent discrimination tasks within the contingent classification tasks. These tasks were in no way different from the control classification task.

\section{Discussion}

As for letters in Experiment 3, the use of color as the cuing dimension for lines gave no evidence of effective contingent processing, unlike the use of location as the cuing dimension for lines in Experiment 2. In Experiment 3 , the entire surrounding field was colored to provide the cuing dimension. In this experiment, the lines themselves were colored because of the possibility that it was easy to ignore color as the cuing dimension when it surrounded the contingent dimension, but that it could not be ignored when it was part of the stimulus itself. The results make it clear that neither method of presenting the cuing dimension of color was effective. Furthermore, there was a 90-msec difference between RT for the control classification and the mean of the two pairs of discrimination tasks, so there was ample opportunity for the contingent classification task to provide improvement. That no such improvement occurred once again suggests that the failure of effective contingent processing is due to the nature of color itself as a cuing dimension.

\section{GENERAL DISCUSSION}

The primary purpose of these experiments was to investigate contingent classification when the contingent stimuli were much more difficult to identify and discriminate than those used by Garner (1985). The reason for doing this was the assumption that subjects would be willing to allocate more attention to the cuing dimension if the contingent dimension was sufficiently difficult. Letter stimuli used by Garner (1985) were made more difficult, and line stimuli were also used because they are more difficult than the easily perceived letter stimuli used by Garner (1985). A secondary purpose, however, was to determine whether the two cuing dimensions of location and color were equally effective in specifying the contingency.

The results were clear in that contingent classification was effective with the more difficult stimuli; however, it also is clear that this effectiveness was obtained only with location as the cuing dimension. Color had been chosen in addition to location because of evidence that color has some special status compared to dimensions such as brightness, size, and form (see Garner, 1974, 1983, 
for a summary of this evidence). One task that has similarities to the contingent classification task is the partial report procedure of the kind used to establish the existence of an iconic store. Both Sperling (1960) and Averbach and Coriell (1961), in the original experiments on iconic memory, used location to specify what was to be reported. Von Wright (1968) later used several other dimensions, and found that color was essentially as good as location in specifying the subset of stimuli to report. So there was good reason to expect that location and color could both be effective cuing dimensions in contingent classification.

\section{Cuing Dimensions as Channels}

Garner $(1974,1983)$ pointed out that the concept of a channel (i.e., a property of a stimulus that makes information available, but that is not an informational aspect of the stimulus) was necessary in distinguishing some stimulus dimensions from others. Thus a modality is a channel, because it makes information available, but does not itself constitute information. The role of channel, of course, is exactly what is required of the cuing dimension, each level being a channel that makes information available but that is not itself an informational aspect of the stimulus: It provides no information about what response should be used.

Perhaps this is the basic difference between color and location: color is unable to function as a channel, whereas location is by its nature a channel almost as much as it is a modality. Several authors have mentioned this special status of visual location. Duncan (1981) noted that advance knowledge of position is especially effective in providing selective attention, and in fact much of the current research on selective attention simply assumes that location is the appropriate dimension upon which to base selective attention. Tsal (1983) made the statement stronger in noting that location information, unlike color, is always present and is thus a special dimension. Kubovy (1981) called location the indispensable dimension for vision. More recently still, Nissen (1985) showed that location information is necessary when other information about an object is to be reported. Thus all of these writers argued that location is very different from color, and even though color may be a strong dimension for search and other selective attention tasks, it does not provide the role of channel, as does location. If so, then the conclusion must be drawn that contingent classification can be effective only if the cuing dimension can function as a channel.

\section{Discriminability of Cuing Dimensions}

Despite this considerable evidence that location is an inherently special dimension in that it can provide a channel, and that color cannot, it is possible that the two cuing dimensions used here were not of equal discriminability, and that this factor influenced the difference in results obtained with the two cuing dimensions. The selections of the particular levels on each dimension were originally made on quite different bases. In the case of color, two colors were used that were as dissimilar as possible so that any failure to obtain effective contingent processing would not be due to a poor choice of specific colors. In the case of location, the primary consideration was to have the locations as different as possible while maintaining foveal vision. Thus the possible loss of acuity with locations too far apart, rather than discriminability per se, was the primary consideration in the choice of specific locations. In selecting levels for both cuing dimensions, the intent was to maximize the possibility of obtaining evidence of contingent processing.

However, the two dimensions do differ in RT when a simple discrimination task is used for the two levels of each dimension. A simple experiment was run with two tasks, one in which the two locations of the letter $O$ from Experiment 1 were used and discrimination of the locations was required, and the other in which the same $O$ stimuli from Experiment 3 were used, but discrimination of the colors was required. The procedure was the same as that for discrimination tasks in Experiment 1. Ten subjects were used, and the two tasks were simply alternated five times through a session of $1 \mathrm{~h}$. The mean RT for the last three replications was $354 \mathrm{msec}$ for color and 305 msec for location $[t(9)=6.65, p<.001]$. (All 10 subjects showed greater speed for location.) Thus it is at least possible to argue that location was effective in providing contingent classification, whereas color was not, only because of the greater discriminability of location.

It can also be argued, however, that what makes location special is the very fact that such highly discriminable differences can be obtained with that dimension. Color simply cannot be made more discriminable, so there is no possible way in which color can serve as an effective cuing dimension if discriminability alone can produce it. There is simply no more discriminability available in the color dimension. Location differences can be decreased or increased, and there might be resultant changes in discriminability that would decrease or increase the effectiveness of location as a cuing dimension. Certainly one limit is that if the two locations are not themselves discriminable, then location cannot function as a cuing dimension.

To summarize, if discriminability is the factor making contingent processing possible, then location must still be considered a special dimension because only with it can discriminabilities great enough to provide contingent processing be obtained. Location is special, and that special status is probably due to its ability to function as a channel, which has the inherent ability to make different locations highly discriminable.

\section{Contingent Stimulus Difficulty}

In making this distinction between dimensions that can and those that cannot provide effective contingent cuing, it should not be forgotten that even location became effective as a cuing dimension only when the contingent stimuli were made very difficult to perceive. But it should be noted that in making the letter stimuli more difficult 
to perceive, the error rate was still quite low (specifically, $4.4 \%$ for Experiment 1, with location-cued letters), and that RT was used as the primary dependent variable. It is possible that if stimuli were made even more difficult to perceive, so that error rate, rather than RT, became the primary or sole dependent variable, color or even other properties such as brightness and size might be effective as cuing dimensions. In other words, it may be that in keeping the task easy enough to use RT as the dependent variable, the full effectiveness of contingent relations may not be available.

\section{REFERENCES}

Averbach, E., \& Coriell, A. S. (1961). Short-term memory in vision. Bell System Technical Journal, 40, 309-328.

Biederman, I. (1972). Human performance in contingent informationprocessing tasks. Journal of Experimental $P_{\text {sychology, 93, 219-238. }}$

Duncan, J. (1981). Directing attention in the visual field. Perception \& Psychophysics, 30, 90-93.

GARNER, W. R. (1974). Attention: The processing of multiple sources of information. In E. C. Carterette \& M. P. Friedman (Eds.), Handbook of perception (Vol. 2, pp. 23-59). New York: Academic Press.

GARNER, W. R. (1983). Asymmetric interactions of stimulus dimensions in perceptual information processing. In T. J. Tighe \& B. E. Shepp (Eds.), Perception, cognition, and development: Interactional analyses (pp. 1-38). Hillsdale, NJ: Erlbaum.

GARNER, W. R. (1985). Contingent information processing: Contingent and precued classification. Perception \& Psychophysics, 38, 237-248.

KUBOVY, M. (1981). Concurrent-pitch segregation and the theory of indispensable attributes. In M. Kubovy \& J. R. Pomerantz (Eds.), Perceptual organization (pp. 55-98). Hillsdale, NJ: Erlbaum.

LaBerge, D., VAN Gelder, P., \& Yellott, J. (1970). A cueing technique in choice reaction time. Perception \& Psychophysics, 7, 57-62.
Lasaga, M. I., \& Garner, W. R. (1983). The effect of line orientation on various information processing tasks. Journal of Experimental Psychology: Human Perception \& Performance, 9, 215-225.

MILLER, J. (1982). Discrete versus continuous stage models of human information processing: In search of partial output. Journal of Experimental Psychology: Human Perception \& Performance, 8, 273-296.

Miller, J. (1983). Can response preparation begin before stimulus recognition finishes? Joumal of Experimental Psychology: Human Perception \& Performance, 9, 161-182.

MILLER, J. (1985). A hand advantage in preparation of simple keypress responses: Reply to Reeve and Proctor (1984). Joumal of Experimental Psychology: Human Perception \& Performance, 10, 541-553.

NissEN, M. J. (1985). Accessing features and objects: Is location special? In M. I. Posner \& O. S. M. Marin (Eds.), Attention and performance XI (pp. 205-219). Hillsdale, NJ: Erlbaum.

PoSNer, M. I. (1978). Chronometric explorations of mind. Hillsdale, NJ: Erlbaum.

Reeve, T. G., \& Proctor, R. W. (1984). On the advance preparation of discrete finger responses. Journal of Experimental Psychology: Human Perception \& Performance, 10, 541-553.

ReEve, T. G., Proctor, R. W. (1985). Nonmotoric translation processes in the preparation of discrete finger responses: A rebuttal of Miller's (1985) analysis. Journal of Experimental Psychology: Human Perception \& Performance, 11, 234-241.

ShafFer, L. H. (1965). Choice reaction time with variable S-R mapping. Journal of Experimental Psychology, 70, 284-288.

SPERLING, G. (1960). The information available in brief presentations. Psychological Monographs, 74, 11(Whole No. 498).

TsaL, Y. (1983), On interpreting the effects of location preknowledge: A critique of Duncan. Perception \& Psychophysics, 34, 297-298.

VON WRIGHT, J. M. (1968). Selection in visual immediate memory. Quarterly Journal of Experimental Psychology, 20, 62-68.

WINER, B. J. (1971). Statistical principles in experimental design. New York: McGraw-Hill.

(Manuscript received August 18, 1986; revision accepted for publication December 8, 1986.) 